\title{
The HI that Barked in the Night
}

\section{Mike Disney}

School of Physics and Astronomy, Cardiff University, Queen's Buildings, 5 The Parade, Cardiff CF24 3YB, Wales, UK.

\begin{abstract}
I discuss some of the interesting discoveries gradually emerging from the HIPASS HI survey. Why were so very few dark clouds and dark galaxies identified? Could that be partly due to optical misidentifications? In some cases yes. Will Arecibo overcome some of the deficiencies of HIPASS? I argue not because large telescopes are ill suited to blind surveys. I discuss the problem of Inchoate Galaxies which can be neither young nor old, and the constancy of HI column density found amongst all sources turning up in blind HI surveys. Could some of these unexpected phenomena be the result of Spin Temperature Freezeout? If so there is a lot more HI out there than we imagined.
\end{abstract}

Keywords. Galaxies: formation, Cosmology: dark matter

\section{Introduction}

It's almost exactly 10 years since HIPASS saw first light and this seems a suitable moment to look back and reflect on what it has taught us so far and to ask what we have still to learn. Considering that it was three orders of magnitude faster than its predecessors at making blind HI surveys it will not be surprising if it takes us quite some time to digest some of its most important results. Not the least of these are its negative results, such as its failure to discover the large number of $\mathrm{HI}$ clouds un-associated with optical counterparts which we had anticipated when we began. Of 4000 plus southern HIPASS sources not one appears to be lacking a plausible optical counterpart (Doyle et al. 2006). Hence my title. Has all the intergalactic HI really produced stars - which seemed very unlikely to us when we began - or are there subtler forces at work? Are Dark Galaxies containing HI really absent from the universe or could we be fooling ourselves? And will successor multibeam receivers, fitted to larger telescopes like Arecibo, render HIPASS obsolete? My theme is that caution and thoughtfulness should be our watchwords for now. Particularly so when optical follow up observations to very few HIPASS sources have been published so far.

\section{The identification of optical counterparts in HI surveys}

Because we have radial velocities as well as positions it is all too easy to convince oneself that a bright galaxy near the HI position, which has just the right optical velocity as well, is in truth the source of the HI emission - when it is not. Dont forget that galaxies are strongly clustered in redshift space too. And if Intergalactic Gas Clouds (IGCs) and Dark Galaxies (DGs) are clustered with visible ones, as seems plausible, they will generally have bright companions of the 'right' radial velocity. A cautionary tale here was the early claim by QSOAL astronomers who found a bright galaxy of the right radial velocity associated with every low redshift absorption system. Subsequent careful follow up work has shown, in most cases, that insignificant dwarfs and Low Surface Brightness Galaxies, clustered with the bright galaxies, were actually responsible. 


\begin{tabular}{c|c} 
Position off-set $d \Delta \Theta(\mathrm{kpc})$ & Probability of finding a random galaxy \\
\hline 50 & 0.8 \\
30 & 0.4 \\
10 & 0.1
\end{tabular}

Table 1. The probablity of finding galaxies at different position off-sets.

We shall now estimate the probability of finding a random optical galaxy within a given distance, in both angular and redshift space, of any given HI source. We shall assume, as the observations clearly suggest, that optical galaxies and HI sources are clustered together. For a HIPASS source the acceptable volume $\left(V_{a c c}\right)$ in which an optical counterpart could lie is a long thin cylinder, centred on the source, with its long axis, set by the radial-velocity uncertainties, along the line of sight. For a source at a typical radial velocity $\approx 2000 \mathrm{~km} \mathrm{~s}^{-1}$ the angular uncertainties in position (up to 5 arcmins) correspond to $\approx 50 \mathrm{kpc}$, while the velocity uncertainties $\Delta V$ amount to $H_{O} \Delta V(\approx 30 \mathrm{~km}$ $\mathrm{s}^{-1}$ ) or half a Mpc. Given the correlation function:

$$
p(r) d V=n_{0} d V(1+\xi(r))
$$

where $\xi(r)=\left(r / r_{0}\right)^{1.8}$ and $n_{0}$ is the average number of plausible galaxies per $\mathrm{Mpc}^{-3}$, it is possible to integrate the probability of finding a random galaxy within the volume $V_{a c c}$ of the acceptable cylinder. To a very good approximation the number within an angular distance $\Delta \theta$ of the source, at distance $d$, is given by:

$$
N[<d(M p c) \delta \theta(\text { rads })] \approx 1.8 n_{0} r_{0}^{1.8}(d \delta \theta)^{1.2}
$$

where $r_{0} \approx 8 \mathrm{Mpc}$. Notice that the number is only weakly dependent on $\delta \theta$ (because of the strong correlation) and dependent on the radial velocity uncertainty not at all. This last is counter-intuitive but arises from the long thin shape of the cylinder. The ends of the cylinder are so very far from the centre that finding highly clustered galaxies within the ends is very unlikely.

To turn equation 2.2 into numbers it is necessary to adopt an optical Luminosity Function for the putative galaxies.

If we adopt the Blanton et al., ( 2003) LF, and if we are prepared to accept as our identification an optical galaxy up to 3 mags below $\mathrm{M}^{*}$ then Table 1 gives the probability of finding such a random galaxy within an angular size distance $\Delta \Theta$ (radians) of the 21-cm source, where $d$ is the distance of the source away from us. Now the positional uncertainty for radio centroids in HIPASS is typically 1.3 arc mins, (Zwaan et al. 2004 and Meyer et al. 2004 ) which at a typical source distance of $2000 \mathrm{~km} \mathrm{~s}^{-1}$ corresponds to a $d \Delta \Theta$ of $10 \mathrm{kpc}$. But sources are sometimes identified up to 5 (Doyle et al. 2006) and even 7 (Wong et al. 2005) arc mins away from the radio centroids. It must be clear from Table 1 that the possibility of misidentifying an IGC or a DG with a plausible optical galaxy must be rather high and that there may still be many such hidden in the HIPASS catalogues. The obvious question then is: 'Will the new surveys with Arecibo, with its much improved sensitivity and resolution, overcome the difficulties of HIPASS?'. The answer, to the surprise of many, is 'No'. Why not? Because bigger telescopes find the bulk of their sources at a correspondingly larger distance away where they lose their advantages in angular resolution, beam filling and sensitivity. In other words they will simply find the same sources with the same problems, but further away. 


\section{Why big telescopes are ill suited to blind HI surveys}

The fact that a big dish is undoubtedly so much better for examining a source already known cannot be used to argue that it is equally better for making blind surveys. It has to pay two prices for its smaller beam: (i) less sky coverage/unit time (obv.) and (ii) a noisier sky/unit area (see below). The first limits its volume coverage for sources of a given $M_{H I}$, the second its column-density sensitivity (more subtle). The 3 governing equations are:

$$
d_{\max }\left(M_{H I}\right) \approx M_{H I}^{1 / 2} D t^{1 / 4}
$$

where $D$ is the dish diameter, $d$ the distance and $t$ the integ.time/beam which is obvious, given that system noise dominates and is independent of $\mathrm{D}$. The survey speed is given by:

$$
\frac{\operatorname{Vol}\left(M_{H I}\right)}{T} \approx M_{H I}^{3 / 2} D t^{-1 / 4} N_{b}
$$

where $N_{b}=$ No.of multibeams/tel. This equation follows from equation 3.1. Note the temptation to use short integration times to increase the number of sources found. There being no such thing as a free lunch, there is a price to pay however, a price which follows from the next, more subtle, equation for the column density sensitivity:

$$
N_{H I} \approx t^{-1 / 2}
$$

Equation 3.3 is independent of D, which is seldom acknowledged by experienced HI observers, who apparently seem to believe in free lunches. It follows (see below) because larger tels. project the same system noise into smaller beams, and hence have to work against an apparently noisier sky.

As an example we can compare the Arecibo blind surveys ALFALFA and AGES against HIPASS.

(1) ALFALFA maximises the source-finding speed by reducing $t$ per beam: Speed $\frac{\text { ALFALFA }}{\text { HIPASS }} \propto D t^{-1 / 4} N_{b}=\frac{305}{64} \times \frac{28 s e c}{450 \mathrm{sec}}^{-1 / 4} \times \frac{7}{13} \approx 5$ times faster for a given $M_{H I}$. Its survey depth $d_{\max }$ (see eq. 3.1) is 2.4 times greater than HIPASS so the number of sources it will find/unit area will be $(2.4)^{3}=14$ times greater. However the sky coverage (as a fraction of the total) is 0.23 times less, so the total number of sources found of a given $M_{H I}$ (e.g. low mass clouds) will only be $14 \times 0.23=3.2$ times greater, which is hardly significant. Worse still, because of its low integration time/beam $(28 \mathrm{sec})$ its column density sensitivity (see eqn. 3.3) will be 4 times worse than HIPASS, making it unsuited to searching for such clouds anyway. Since its typical sources will be 2.4 times further away it has a slight resolution advantage over HIPASS of $[305 /(64 \times 2.4)]=2$ times better, which will help with source identification. Altogether though it is hard to see how ALFALFA will afford any significant scientific advance beyond HIPASS.

(2) AGES maintains the same $N_{H I}$ (i.e. surface brightness) sensitivity as HIPASS by using comparable integration times. Its survey speed is then $\propto D N_{b}=\frac{305}{64} \times \frac{7}{13}=2.5$ times faster but its typical sources are $305 / 64=4.75$ times further away. However since it is targeted (unlike ALFALFA) at specific targets of known redshift (e.g. clusters), it does have 305/64 times better physical resolution at that redshift and it can use unused correlator capacity to obtain higher velocity resolution, which can be useful for finding narrow-line sources $(t \rightarrow t \Delta v$ in many of the above equations because of the Bandwidth Theorem). Again though one cannot expect dramatic improvements over HIPASS. My point is not to criticize these surveys but to plead that much of the time at Arecibo 


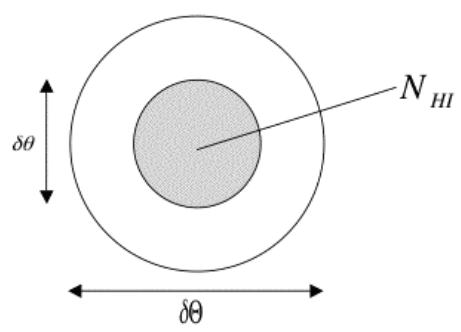

Figure 1. A source contained within the beam

should be assigned to following up sources found with smaller telescopes, at which it is still supreme.

Column Density - We next derive the No Free Lunch theorem; i.e. while you find more sources with shorter integrations you must lose Column-Density-sensitivity to pay for it. For a source inside the telescope beam (see figure 1):

$$
\begin{aligned}
\text { Signal } & \approx N_{H I}(\delta \theta)^{2} t \\
\text { Noise } & \approx k T_{\text {sys }} t^{1 / 2}
\end{aligned}
$$

If we fix $T_{s y s}$ and $S / N$ then for detection $N_{H I} \geqslant \frac{D^{2} t^{1 / 2}}{(\delta \theta)^{2}}$. So the bigger the source obviously the lower the column density can be. In the limit: as $\delta \theta \rightarrow \delta \Theta \sim 1 / D$ so $N_{H I} \sim t^{-1 / 2}$ which is independent of $D$ ! At first sight this looks paradoxical. Why has the big telescope lost its advantage? Answer: because (i) system noise dominates and is independent of telescope size and (ii) the big telescope projects that same noise onto a much smaller area of sky (its beam). So a big dish is looking at an artificially bright sky, one brighter than its smaller cousin by a factor $D$ squared! This exactly cancels its advantage in area.

Beam filling - experienced observers immediately retort "But a source in a bigger telescope fills more of the beam". True, IF the source had a fixed angular size. But look at the survey equation 3.1: IN A BLIND SURVEY A BIG TELESCOPE FINDS ITS SOURCES $D$ TIMES FURTHER AWAY and therefore they are $D$ times smaller in angular size. So beam filling is identical for comparable sources! These matters are subtle so we should not be surprised to find that they are not widely understood. In consequence many claims as to very low values of the cosmic $\Omega_{H I}$ and the cosmic $\Omega_{L S B G}$ in truth refer only to high column density material and are therefore not very interesting and their significance is exaggerated. In general, column-density sensitivity should not be claimed a priori, but measured retrospectively from the weakest sources in the survey. [As an example the claimed sensitivity of AHISS (Zwaan et al. 97) was completely ill founded, as was the implication that LSBGs must be very rare.]

\section{Dark galaxies at $21 \mathrm{~cm}$}

$21 \mathrm{~cm}$ observers could render astronomy a great service by revealing the presence of Dark Galaxies - if they exist. Jon Davies will discuss this subject but I do want to make one simple point. Are there clear ways to distinguish between DGs and tidal debris? Yes there can be - if you are lucky. One doesn't have to do elaborate simulations to convince oneself that in order to observe radial velocity changes of galaxy size within distances of galaxy scale (as in the case of VIRGOHI21) one needs to have galaxy masses close to 
the line of sight. In this case there are none visible so the perturbing mass must be dark. Although many referees have tried, none has been able to overthrow this simple argument. And although many experienced observers have claimed to have found sources just like VIRGOHI21 none, on closer inspection, appears to be anything like it in its combination of large line-width and small physical size. Whatever else it is VIRGOHI21 is not tidal debris! Those who doubt should read the detailed argument in Minchin et al. 2007.

\section{The problem of Inchoate Galaxies}

We have recently completed a cross-correlation of several hundred HIPASS sources with the overlapping SDSS (Appadoo et al. 2007). The sources are very diverse and range from 'Hydrogen Giants' $\left(M_{H I}>10^{10} M_{\odot}\right)$, through massive LSBGs $\left(M_{d y n}>10^{11}\right.$ $M_{\odot}$; SB $>1.5$ mag. dimmer than the Freeman value) to 'Inchoate Galaxies' (below), though the majority are late type gas-rich spirals 1 to 2 mags fainter than $\mathrm{M}^{*}$.

'Inchoates' are extremely low SB objects, barely visible in the SDSS, with no obvious visible cohesion - simply dim patches of unconnected light. They have $\left(M_{H I} / L_{B}\right) \mathrm{s}$ of 5 or more in solar units and would not turn up except in blind HI surveys. The prototype is the Giovanelli and Haynes object (1989) which is one of at least half a dozen in this survey. The Inchoates present a real problem (see also Salzer et al. 1991), because they can't be young, but they can't be old either. If they were young then where are the progenitor pure HI clouds? And if they were old, but fading after a recent starburst, they are so dim already that they would quickly become invisible, leaving behind dark HI clouds - which again appear to be rare. The only way I can think of resolving this dilemma is if the young stars in such objects can switch their HI emission 'on and off' which is not impossible - see section 7 below.

\section{Constant HI column density}

Minchin (2003) and Rosenberg et al., (2005) have independently discovered a remarkable regularity among HI sources turning up in blind HI surveys. To within the measurement errors they all have the same column density of $10^{20.65 \pm 0.3}$ atoms $\mathrm{cm}^{-2}$. It is distance independent and reminiscent of the constant surface brightness law for discs first discovered by Holmberg (1965) and highlighted by Freeman (1970). It is not a selection effect, see Minchin et al. (2003); and it needs explaining. The obvious hypothesis is that higher col. density material is transformed into stars whilst lower column density gas is ionised by the IGRF e.g. Linder et al. (2003). However that obviously requires a strong coincidence. Another - see section 7 below - is that lower column density gas is unexcited and so cannot emit.

\section{Spin temperature freeze out}

To emit $21 \mathrm{~cm}$. radiation the upper hyperfine state of HI must be continuously reoccupied. At high enough densities collisional excitation will suffice. At intermediate densities $\mathrm{Ly}_{\alpha}$ photons scattering many times as they escape from the gas will leave a trail of excited HI atoms - the so called Barkhausen-Field effect (e.g. Kulkarni and Heiles 1988). But modern equipment such as the Parkes multibeam is capable of detecting lower and lower column densities. At a low enough column density a Ly $\mathrm{L}_{\alpha}$ photon will quickly escape, scattering too seldom to excite many atoms into the upper $21-\mathrm{cm}$. level. The 'frozen' gas will not emit at $21 \mathrm{~cm}$ though it will still be detectable in absorption against background 
UV sources such as QSOs. Watson and Deguchi (1984) simulated the escape of Ly pho- $_{\alpha}$ tons - excited by intergalactic X-rays, from a plane parallel layer of HI. They found rapid escape, i.e. 'Spin Temperature Freeze Out' at just below the $N_{H I} / \Delta V$ levels detected by Minchin and Rosenberg. Could this be just coincidence? Note that such freeze-out could also account for the oddity of the Inchoates. When they form a small burst of blue stars the $\mathrm{Ly}_{\alpha}$ photons there might unfreeze the surrounding HI, which then begins to emit at $21 \mathrm{~cm}$. But when the blue stars fade the HI freezes back again on a timescale of $10^{7}$ years and returns to its inert state. If Spin temperature Freeze Out is widely prevalent then there could be a lot more HI out there than we presently imagine (Disney and Minchin 2003).

\section{Acknowledgements}

We all owe an enormous debt to some visionary Australian astronomers, notably Ron Ekers and Lister Stavely-Smith who saw the potential of multi-beam receivers at $21 \mathrm{~cm}$. back in the early 1990s and built the Multibeam system which has been so successful. It's main results have still to emerge.

\section{References}

Appadoo D. G. West A. et al., 2007, MNRAS submitted

Blanton M. R. et al., 2003, ApJ, 592, 819

Disney M. J., \& Minchin R. F., 2003, astro-ph/0211288

Doyle M. T., \& Drinkwater M. J., 2006, MNRAS, 372, 977

Freeman K. C., 1970, ApJ, 160, 811

Giovanelli R., \& Haynes M. P., 1989, ApJL, 346, 1989

Holmberg E., 1965, ArA, 3, 387

Kulkarni S. R., \& Heiles C., 1988, in Galactic and extragalactic radio astronomy, ed. K. I. Kellerman and G. L. Verschuur (2d ed.; Berlin:Springer), 95

Linder S., et al., 2003,MNRAS,

Meyer M. J. et al., 2004, MNRAS, 350, 1195

Minchin, R. F. 2003, PhD thesis, Cardiff University

Minchin R. F. et al., 2003, MNRAS, 346, 787

Minchin, R. F. et al., 2007, ApJ, accepted

Rosenberg J. L., Schneider S.E., \& Posson-Brown J., 2005,ApJS, 129, 1311

Salzer J. J., di Serego Alighieri S., Matteucci F., Giovanelli R., \& Haynes M., 1991, AJ, 101, 1258

Watson W. D., \& Deguchi, S., 1984,ApJ, 281,L5

Wong O. I. et al., 2005, MNRAS, 371, 1855

Zwaan M. A., Briggs F. H., Sprayberry D., \& Sorar E., 1997, ApJ, 490, 173

Zwaan M. A. et al., 2004, MNRAS, 350, 1210 